OPEN ACCESS

Edited by:

Pierfrancesco Franco,

University of Eastern Piedmont, Italy

Reviewed by:

Luca Boldrini,

Dipartimento di Radioterapia Oncologica, Fondazione Policlinico A.

Gemelli IRCCS, Italy

Luciana Caravatta,

SS Annunziata Polyclinic Hospital,

Chieti, Italy

*Correspondence:

Xian Liu

liuxian74@hotmail.com

Specialty section: This article was submitted to

Cancer Imaging and

Image-directed Interventions,

a section of the journal

Frontiers in Oncology

Received: 21 April 2021

Accepted: 03 June 2021

Published: 01 July 2021

Citation:

Chen W, Mao L, Li L, Wei Q, Hu S, Ye Y, Feng J, Liu B and Liu X (2021)

Predicting Treatment Response of Neoadjuvant Chemoradiotherapy in Locally Advanced Rectal Cancer Using Amide Proton Transfer MRI Combined With Diffusion-Weighted Imaging.

Front. Oncol. 11:698427.

doi: 10.3389/fonc.2021.698427

\section{Predicting Treatment Response of Neoadjuvant Chemoradiotherapy in Locally Advanced Rectal Cancer Using Amide Proton Transfer MRI Combined With Diffusion-Weighted Imaging}

\author{
Weicui Chen ${ }^{1}$, Liting Mao ${ }^{1}$, Ling $\mathrm{Li}^{1}$, Qiurong Wei ${ }^{1}$, Shaowei $\mathrm{Hu}^{2}$, Yongsong $\mathrm{Ye}^{1}$, \\ Jieping Feng ${ }^{1}$, Bo Liu ${ }^{1}$ and Xian Liu ${ }^{1 *}$ \\ 1 Department of Radiology, The Second Affiliated Hospital, Guangzhou University of Chinese Medicine, Guangzhou, China, \\ 2 Department of Pathology, The Second Affiliated Hospital, Guangzhou University of Chinese Medicine, Guangzhou, China
}

Objective: To evaluate amide proton weighted (APTw) MRI combined with diffusionweighted imaging (DWI) in predicting neoadjuvant chemoradiotherapy (NCRT) response in patients with locally advanced rectal cancer (LARC).

Methods: 53 patients with LARC were enrolled in this retrospective study. MR examination including APTw MRI and DWI was performed before and after NCRT. APTw SI, ADC value, tumor size, CEA level before and after NCRT were assessed. The difference of the above parameters between before and after NCRT was calculated. The tumor regression grading (TRG) was assessed by American Joint Committee on Cancer's Cancer Staging Manual AJCC 8th score. The Shapiro-Wilk test, paired t-test and Wilcoxon Signed Ranks test, two-sample t-test, Mann-Whitney $U$ test and multivariate analysis were used for statistical analysis.

Results: Of the 53 patients, 19 had good responses (TRG 0-1), 34 had poor responses (TRG 2-3). After NCRT, all the rectal tumors demonstrated decreased APT values, increased ADC values, reduced tumor volumes and CEA levels (all $p<0.001$ ). Good responders demonstrated higher pre-APT values, higher $\triangle$ APT values, lower pre- ADC values and higher $\Delta$ tumor volumes than poor responders. Pre-APT combined with preADC achieved the best diagnostic performance, with AUC of 0.895 (sensitivity of 85.29\%, specificity of $89.47 \%, p<0.001$ ) in predicting good response to NCRT.

Conclusion: The combination of APTw and DWI may serve as a noninvasive biomarker for evaluating and identifying response to NCRT in LARC patients.

Keywords: Amide proton transfer, diffusion-weighted imaging, neoadjuvant chemoradiotherapy, locally advanced rectal cancer (LARC), treatment response 


\section{HIGHLIGHTS}

- After NCRT, All the rectal tumors demonstrated decrease APT values, increased ADC values, reduced tumor volumes and CEA levels.

- Good responders to NCRT demonstrated higher pre-APT values, higher $\Delta \mathrm{APT}$ values, lower pre-ADC values and higher $\Delta$ tumor volumes than poor responders.

- A combination of APT and ADC values before NCRT exhibited a good diagnostic performance in predicting a good response to NCRT (with AUC of 0.895, sensitivity of $85.29 \%$ and specificity of $89.47 \%$ ).

\section{INTRODUCTION}

Currently, preoperative neoadjuvant chemoradiotherapy (NCRT) followed by total mesorectal excision surgery is the standard treatment protocol for locally advanced rectal cancer (LARC) (1). NCRT aims to downstage the tumor, enable complete surgical resection, and reduce the risk of recurrence and metastases (2). Some strictly selected patients can even achieve complete clinical response with a "wait and see" policy after NCRT, avoiding surgical treatment (3). However, significant unexplained variation remains in the responses to NCRT. A series of clinical trials demonstrated that $8 \%$ to $27.5 \%$ of patients who achieved pathologic complete response (pCR) after NCRT have a better long-term outcome, lower recurrence risk, and improved overall survival (4). Approximately $54-75 \%$ of patients had tumor downstaging, and the remainder had no treatment response. Therefore, predicting the response to NCRT is important for patients with potentially curable LARC who wish to explore personalized treatment to improve their therapeutic outcomes.

MRI plays an important role in the therapeutic assessment of rectal carcinoma, particularly beneficial to surgical planning and optimize treatment strategies for patients with different responses (5). MR-based tumor regression grade (mrTRG) was validated to be associated with disease-free and survival outcomes by The MRI and Rectal Cancer European Equivalence (MERCURY) trial (6). However, conventional T2WI MRI is limited by its inability to differentiate posttherapeutic edema and fibrosis from residual tumor tissue. Morphological parameters were also proved to be helpful in assessing pCR. Some studies demonstrated a significant correlation between tumor volume reduction and pCR (7-9). Furthermore, Fiorino $\mathrm{C}$ et al. introduced an early regression

\footnotetext{
Abbreviations: ADC, apparent diffusion coefficient; APT, amide proton transfer; AUC, area under the curve; CEST, chemical exchange saturation transfer; DCE, dynamic contrast enhanced; DWI, diffusion weighted; LARC, locally advanced rectal cancer; NCRT, neoadjuvant chemoradiotherapy; ROC, receiver operating characteristic; ROI, region of interest; SD, standard deviation; $\mathrm{pCR}$, pathologic complete response; TRG, tumor regression grade.
}

index based on a logarithmic transformation of change in tumor volume (10). This new predictive index showed great discriminative power in evaluating tumor response to NCRT and long-term disease-free survival $(10,11)$. Functional MRI, such as diffusion-weighted imaging (DWI) and dynamiccontrast-enhanced MRI (DCE-MRI) can provide additional physiological information about a tumor's cellular environment and perfusion characteristics, offering great potential to assess the therapeutic response of rectal cancer (12-15). DWI has been widely used in the evaluation of tumor response to NCRT in rectal cancer, as its capability of providing information on tumor cellular architecture. However, results regarding the use of ADC in predicting the NCRT response have been inconsistent. This variation may be due to a lack of standardized imaging, acquisition techniques, and analysis methods $(5,12)$.

Lately, considerable progress has been made in devising radiomics or deep learning techniques to assess the treatment response of NCRT in LARC $(13,16,17)$. Horvat et al. found that radiomics provided a significantly greater diagnostic capability than T2WI or DWI alone when using a random forest classifier to investigate T2WI-based radiomics while evaluating complete clinical response in rectal cancer patients after NCRT (17). Zhang et al. used a deep learning model based on diffusion kurtosis MRI to predict pCR in assessing the response of LARC after NCRT. The deep learning model showed good diagnostic performance and aided radiologists in assessing pCR (18). However, these extracted radiomics features depend on image acquisition, reconstruction, and processing methods, which naturally vary among different institutes and operators (19). Their clinical application is restricted by reproducibility and reliability. Besides, the deep learning model is typically too complex to interpret the relationship between extracted properties and tumor biology (20). To our knowledge, a reliable classification system has yet to be developed.

Amide proton transfer-weighted (APTw) MRI is a molecular MRI technique based on chemical exchange saturation transfer (CEST), which is achieved through qualifying the exchange between amide proton (-NH) groups of endogenous mobile proteins/peptides and bulk water (21). The APTw signal is related mainly to the concentration of mobile proteins, making it beneficial for assessing tumor grade and differentiation (22-24). Clinical APTw imaging has also shown promise in tumor monitoring in gliomas. Several studies have reported that APTw imaging helps differentiate between pseudo-progression, radiation necrosis, and tumor recurrence in gliomas (25)-it is superior to conventional MRI contrasts as well as to advanced functional imaging methods such as perfusion (dynamic susceptibility contrast and dynamic-contrast-enhanced) and spectroscopy $(24,26-28)$. However, only one study assessed APTw MRI in evaluating the effect of chemoradiotherapy in LARC (29).

Therefore, considering the characteristics of APTw imaging and DWI, we aimed to investigate the value of APTw imaging combined with DWI for predicting the treatment response of NCRT in patients with LARC. 


\section{MATERIALS AND METHODS}

\section{Participants}

The current study was approved by the Medical Ethics Committee of our hospital (Ref. No. YE2019-274-01) and written informed consent was obtained from each patient before the MR examination. Between February 2019 to May 2021, 88 consecutive patients diagnosed with LARC were included. All the patients received preoperative NCRT followed by TME surgery.

The inclusion criteria were as follows: 1) biopsy-proven rectal non-mucinous adenocarcinoma histologically, 2) LARC (category cT3 or cT4, node-positive status) defined on primary MR staging, 3) treatment consisting of NCRT followed by surgical resection. The exclusion criteria were as follows: 1) recurrent rectal cancer, (b) additional treatment (targeted therapy or immunotherapy), 3) interval between restaging rectal MRI and surgery of more than three months, 4) NCRT was incomplete, 5) poor image quality (included motion artifacts and image distortion from susceptibility effect due to bowel gas).

Figure 1 displays the patient selection flowchart.

\section{NCRT Treatment}

NCRT consisted of 45-50 Gy of radiation delivered in daily doses of 1.5 or2 Gy, five fractions per week, and concomitant chemotherapy. Neoadjuvant chemotherapy regimens were as follows: (1) oxaliplatin $130 \mathrm{mg} / \mathrm{m}^{2}$ (iv gtt, d1) and capecitabine $1000 \mathrm{mg} / \mathrm{m}^{2}$ (per os, bid, d1-14) every three weeks for 6-8 courses (XELOX) in eighteen patients; (2) folinic acid $200 \mathrm{mg} / \mathrm{m}^{2}$ (d1), fluorouracil $400 \mathrm{mg} / \mathrm{m}^{2}$ (d1), fluorouracil $2400 \mathrm{mg} / \mathrm{m}^{2}$ (d1-d2), and oxaliplatin $85 \mathrm{mg} / \mathrm{m}^{2}$ (d1) for 3-7 courses (mFOLFOX6) in ten patients; (3) capecitabine $1250 \mathrm{mg} / \mathrm{m}^{2}$ (per os, bid, d1-d14) for 3-6 courses in 25 patients. Surgery with total mesorectal excision was performed within 6-8 weeks after the completion of NCRT.

\section{MRI Protocol}

All participants received two MRI examinations: the first within one week before NCRT (pre-NCRT MRI) and the second within one week before surgery (post-NCRT MRI).

All MRI examinations were performed on a 3.0T MRI scanner (Ingenia, Philips Healthcare, Best, the Netherlands) using a 32-channel phased-array coil. A glycerin enema was performed before the examination to reduce distortion due to gas in the rectum. At $30 \mathrm{~min}$ before the $\mathrm{MR}$ examination, $5 \mathrm{mg}$ of raceanisodamine hydrochloride was injected intramuscularly to reduce peristaltic movement.

The initial sagittal and axial T2-weighted turbo spin echo (TSE) sequences were performed to determine the location of the rectal tumor. For APTw imaging, we used the Philips product implementation. More specifically, axial APTw images were acquired using a 3D TSE mDIXON sequence. The B1 field strength was $2 \mu \mathrm{T}$; continuous RF saturation pulse train had a duration of 2 s. The entire $z$-spectrum contains nine images acquired at various saturation frequency offsets, including $\pm 3.5, \pm$ $3.42, \pm 3.58$, and $-1560 \mathrm{ppm}$. To enhance the signal-to-noise ratio in the APTw images, three of the Z-spectral images are acquired at +3.5 ppm using different echo shifts on the order of $0.5 \mathrm{~ms}$. This allows us to calculate a B0 field map directly from APTw image acquisition via the mDIXON algorithm. Correction of B0 field homogeneity was achieved by a Lagrange interpolation among the different saturation frequency offsets on a voxel-byvoxel basis. mDIXON was applied to suppress lipid artifacts in APTw images. Other imaging parameters were as follows:

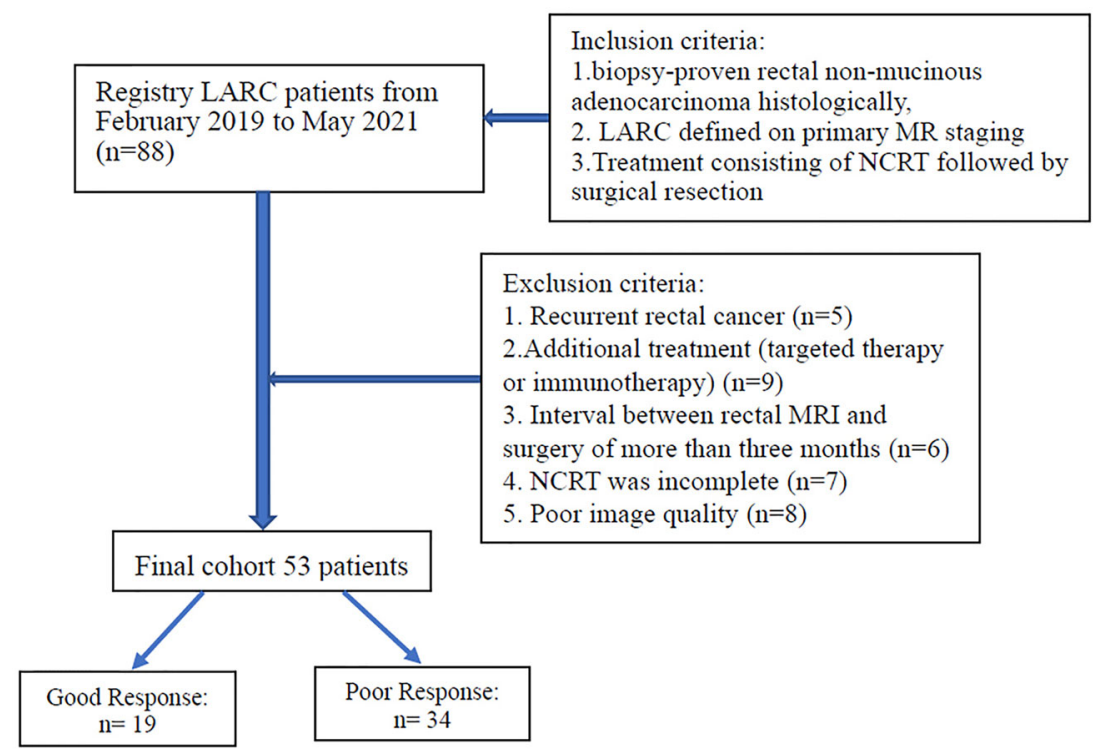

FIGURE 1 | Flowchart of patient selection. 
repetition time (TR)/echo time (TE): $5864 \mathrm{~ms} / 10 \mathrm{~ms}$; field of view (FOV) $250 \times 346$; section thickness $5 \mathrm{~mm}$; voxel size $1.8 \times 1.8 \times$ $5 \mathrm{~mm}$; TSE factor 35 .

Other MR sequences included high-resolution T2-weighted imaging (Turbo SE, TR/TE: 3900/100 ms, flip angle $90^{\circ}$, FOV $200 \times 200 \mathrm{~mm}$, section thickness $3 \mathrm{~mm}$, matrix $288 \times 228$, TSE factor 17) in the axial, coronal, and sagittal planes; conventional axial DWI (echo planar SE, TR/TE $3000 / 72 \mathrm{~ms}$, flip angle $90^{\circ}$, $\mathrm{b}=0,1000 \mathrm{~s} / \mathrm{mm}^{2}$, section thickness $3 \mathrm{~mm}$, matrix $82 \times 82$ ); plain and gadolinium-enhanced T1-weighted (turbo spin echo, TR/TE 578/10 ms, FOV $240 \times 240 \mathrm{~mm}$, section thickness $3 \mathrm{~mm}$, matrix $300 \times 230$ ) in the axial, coronal, and sagittal planes.

\section{Image Analysis}

All raw data were transferred to an Intellispace Portal workstation (Philips Healthcare, Best, the Netherlands). According to the principle of the APT algorithm, APTw signal was defined as the asymmetric magnetization transfer ratio $\left(\mathrm{MTR}_{\mathrm{asym}}\right)$ at $3.5 \mathrm{ppm}$ from the corrected $\mathrm{Z}$ spectrum and displayed as amide proton transfer weighted percentage.

$$
\operatorname{MTR}_{\text {asym }}(3.5 p p m)=\frac{S_{\text {sat }}(-3.5 p p m)-S_{\text {sat }}(+3.5 p p m)}{S_{0}},
$$

where $\mathrm{MTR}_{\text {asym }}[+3.5 \mathrm{ppm}]$ is magnetization transfer ratio (MTR) asymmetry at $+3.5 \mathrm{ppm}$ offset frequency, and $\mathrm{S}_{\text {sat }}$ and $S_{0}$ are the signal intensity acquired with and without selective saturation, respectively.

$$
\text { APTw SI }=\text { MTRasym }[\Delta \omega=+3.5 \mathrm{ppm}](\%) .
$$

The apparent diffusion coefficient (ADC) was calculated from two DWI image sets of different $b$ values $\left(b=0,1000 \mathrm{~s} / \mathrm{mm}^{2}\right)$.

Image analysis was performed in consensus by two radiologists (YY and LM, with 20 and 10 years of experience in rectal cancer MRI, respectively) identified rectal lesions from T2WI together with DWI images. For quantitative analysis of APT SI, MRIcro software was used for manual segmentation of the rectal tumor. The outline of the rectal tumor was drawn manually with a freehand tool on high-resolution T2WI images and defined as the region of interest, avoiding the intestinal cavity. The region of interest was then copied to the corresponding APT image to obtain the average APT SI. The mean APT SI values of all slices were recorded for further analysis.

The changes in APT $(\triangle \mathrm{APT})$ and $\mathrm{ADC}(\triangle \mathrm{ADC})$ values were defined as the difference between the corresponding post and pre-values.

\section{Tumor Volume Evaluation}

Tumor volume was measured before and after NCRT by manually drawing the tumor margin with a PACS system (YLZ Ruitu Information Technology, Guangzhou, China) on T2-weighted images comprising the continuous tumorcontaining image. The whole-tumor volume was then calculated by adding up each cross-sectional volume. Two radiologists (YY and LM) assessed the images in consensus.
Tumor volume reduction ( $\Delta$ tumor volume) was calculated as follows:

$\Delta$ tumor volume $=$ pre-tumor volume - post-tumor volume

\section{Carcinoembryonic Antigen (CEA) Level Evaluation}

Serum CEA levels were measured by the chemiluminescent method. The normal range of CEA is $<5 \mathrm{ng} / \mathrm{ml}$. Serum CEA levels before (pre-CEA) and after NCRT (post-CEA) were assessed approximately one week before CRT and within one week before surgery, respectively. The reduction of CEA was calculated as follows: $\triangle \mathrm{CEA}=$ post-CEA - pre-CEA.

\section{Histological Analysis}

All resected specimens were fixed in buffered formalin, embedded in paraffin and then made into $4-\mu \mathrm{m}$ tissue sections for pathologic diagnosis. A pathological evaluation was performed by one pathologist (HS, with 21 years of experience). The pathologic tumor staging and tumor response to CRT were assessed according to the criteria described in the American Joint Committee on Cancer's Cancer Staging Manual (AJCC $8^{\text {th }}$ edition) (30). The grade of tumor response to CRT was classified into four categories: TRG 0 (complete regression): no residual cancer cells; TRG 1 (near-complete regression): single or small groups of cancer cells; TRG 2 (moderate regression): residual cancer with desmoplastic response; TRG 3 (minimal regression): minimal evidence of tumor response. Patients with TRG $0-1$ were considered to have a good response, whereas those with TRG 2-3 were considered to show a poor response to CRT (30).

\section{Statistical Analysis}

Statistical analysis was performed using SPSS 20.0 (IBM, Armonk, New York) and MedCalc Statistical Software version 19.1.2 (MedCalc Software bv, Ostend, Belgium; https://www. medcalc.org; 2019).

The inter-class correlation coefficient was used to assess interobserver agreement for the measurement of APT values, ADC values, and tumor volume values before and after NCRT. Interclass correlation coefficient estimates above 0.75 were considered to have good reliability.

The Shapiro-Wilk test was used to determine the normality of data distribution. The paired t-test (normal distribution) and Wilcoxon signed-rank test (normality test failed) were used to assess the changes in APT, ADC, tumor volume, and CEA level between pre-NCRT and post-NCRT. A two-sample t-test and Mann-Whitney $U$ test were used to assess the difference in the variances between good responders and poor responders. Logistic regression analysis was used to combine pre-APT and pre-ADC values to build a multi-parametric model. The Hosmer- Lemeshow test was used to measure the goodness- of- fit of the multivariate logistic model, and odds ratio (OR) and 95\% CI was calculated.

Receiver operating characteristic (ROC) curve analysis was performed to evaluate the ability of six MR parameters (pre-APT\& pre-ADC, pre-APT, pre-ADC, $\triangle \mathrm{APT}, \triangle \mathrm{ADC}$ and 
$\Delta$ tumor volume) in discriminating good responders from poor responders. The sensitivity, specificity, positive predictive value (PPV), and negative predictive value (NPV) were calculated. A pairwise comparison of receiver-operating-characteristic curves was applied to test for significant differences between the areas under six receiver-operating-characteristic curves. A statistically significant difference was defined to be $p<0.05$.

\section{RESULTS}

\section{Patient Characteristics}

Eventually, 53 patients (average age, 60.2 years; range, 31-85 years) met the inclusion criteria and were enrolled in this study, consisting of 15 females and 38 males. The distribution of patients' characteristics, including age, sex, histologic grade, TNM stage, and TRG status, is shown in Table 1. Of the 53

TABLE 1 | Clinic pathologic characteristics of enrolled patients.

\begin{tabular}{|c|c|}
\hline Characteristic & Number of Patients \\
\hline Age (y) & $31-85 y(60.2 \pm 12.6 y)^{*}$ \\
\hline \multicolumn{2}{|l|}{ Gender } \\
\hline Male & 38 \\
\hline Female & 15 \\
\hline \multicolumn{2}{|l|}{ Clinical Stage before NCRT } \\
\hline cT3N+ & 26 \\
\hline cT4N0 & 16 \\
\hline cT4N+ & 11 \\
\hline \multicolumn{2}{|l|}{ Histological grade } \\
\hline \multicolumn{2}{|l|}{ G1 (Well differentiated) } \\
\hline G2 (Moderately differentiated) & 45 \\
\hline G3 (Poorly differentiated) & 8 \\
\hline \multicolumn{2}{|l|}{ ypT Stage } \\
\hline $\mathrm{T} 2$ & 10 \\
\hline T3 & 36 \\
\hline T4 & 7 \\
\hline \multicolumn{2}{|l|}{ ypN Stage } \\
\hline No & 16 \\
\hline $\mathrm{N} 1 \mathrm{a}$ & 20 \\
\hline N1b & 11 \\
\hline $\mathrm{N} 1 \mathrm{C}$ & $\mathrm{N} / \mathrm{A}$ \\
\hline $\mathrm{N} 2 \mathrm{a}$ & 6 \\
\hline $\mathrm{N} 2 \mathrm{~b}$ & N/A \\
\hline \multicolumn{2}{|l|}{ Tumor regression grade (TRG) } \\
\hline TRG 0 & 6 \\
\hline TRG 1 & 13 \\
\hline TRG 2 & 27 \\
\hline TRG 3 & 7 \\
\hline Good Responders & 19 \\
\hline Bad Responders & 34 \\
\hline
\end{tabular}

Unless otherwise indicated, data are numbers of patients. *Data are mean \pm standard deviation (range). Staging of tumors and TRG were in accordance with American Joint Committee on Cancer TNM classification. Grading of tumors was based on the WHO grading criteria. patients, 19 patients were defined as good responders (TRG $0-1$ ) and 34 patients as poor responders (TRG 2-3).

\section{Inter-Observer Agreement}

The interclass correlation coefficient of the two observers' measurements were 0.934 (95\% CI: 0.876-0.966) for pre-APT, 0.856 (95\% CI: 0.739-0.923) for post-APT, 0.840 (95\% CI:0.7110.915 ) for pre-ADC, 0.862 (95\% CI: 0.748-0.926) for post-ADC, 0.990 (95\% CI: 0.981-0.995) for pre-volume, and 0.973 (95\% CI 0.946-0.986) for post-volume. The two observers' measurements of APT values, ADC values and tumor volumes showed strong agreement (Table 2).

\section{Comparison of APT, ADC, Tumor Volume, and CEA Level Between Pre- and Post-NCRT}

After NCRT, all the rectal tumors had lower APT values (2.794 \pm 0.575 vs $1.687 \pm 0.527, \mathrm{t}=12.315, \mathrm{p}<0.001)$ and higher ADC values $\left(1.020 \pm 0.10510^{-3} \mathrm{~mm}^{2} / \mathrm{s}\right.$ vs $1.120 \pm 0.11110^{-3}$ $\left.\mathrm{mm}^{2} / \mathrm{s}, \mathrm{t}=-10.475, \mathrm{p}<0.001\right)$. The tumor volume decreased significantly from a median of $31.95 \mathrm{~cm}^{3}$ (range $7.68-115.60 \mathrm{~cm}^{3}$ ) before NCRT to a median of $11.73 \mathrm{~cm}^{3}$ (range $1.25-42.50 \mathrm{~cm}^{3}$ ) after NCRT $(\mathrm{z}=-6.334, \mathrm{p}<0.001)$. A median volume reduction rate of $63.3 \%$ was found. The CEA level decreased significantly from a median of $21.87 \mathrm{ng} / \mathrm{ml}$ (range of 3.26-169.70 ng/ml) before NCRT to a median of $5.30 \mathrm{ng} / \mathrm{ml}$ (range $0.81-63.04 \mathrm{ng} / \mathrm{ml}$ ) after NCRT $(\mathrm{z}=-6.335, \mathrm{p}<0.001)$ (Figure 2).

\section{Parameter Comparison Between Good and Poor Responders to NCRT}

Significant differences were found between good and poor responders for pre-APT values, $\Delta \mathrm{APT}$, pre-ADC, and $\Delta$ tumor volume. The good responder group demonstrated higher preAPT values, higher $\triangle \mathrm{APT}$ values, lower pre-ADC values and higher $\Delta$ tumor volumes than the poor responder group (Figures 3-6). However, no difference was found in post-APT values, post-ADC values, $\triangle \mathrm{ADC}$ values, pre-volumes, post-volumes, pre-CEA levels, post-CEA levels and $\triangle \mathrm{CEA}$ levels between the good and poor responder groups (all p > 0.05) (Table 3).

\section{Diagnostic Capacity of APT and ADC in Predicting NCRT Treatment Response}

The significant level of the Hosmer-Lemeshow test was 0.245 , ORs of pre-APT and pre-ADC were $0.044(\mathrm{p}<0.001), 0.001$ $(p=0.006)$, respectively, suggesting the fit of the model had good goodness.

The sensitivity, specificity, PPV, and NPV of pre-APT combined with pre-ADC, pre-APT, $\triangle \mathrm{APT}$, pre-ADC value, $\triangle \mathrm{ADC}$ and $\Delta$ tumor volume for predicting the response to

TABLE 2 | ICC for APT, ADC and volume values before and after NCRT measured by two radiologists.

\begin{tabular}{|c|c|c|c|c|c|c|}
\hline & Pre-APT & Post-APT & Pre-ADC & Post-ADC & Pre-Volume & Post-Volume \\
\hline ICC (95\% Cl) & $0.934(0.876-0.966)$ & 0.856 (0.739-0.923) & $0.840(0.711-0.915)$ & $0.862(0.748-0.926)$ & 0.990 (0.981-0.995) & $0.973(0.946-0.986)$ \\
\hline
\end{tabular}

ICC, interclass correlation coefficient; 95\% Cl, 95\% confidence interval. 

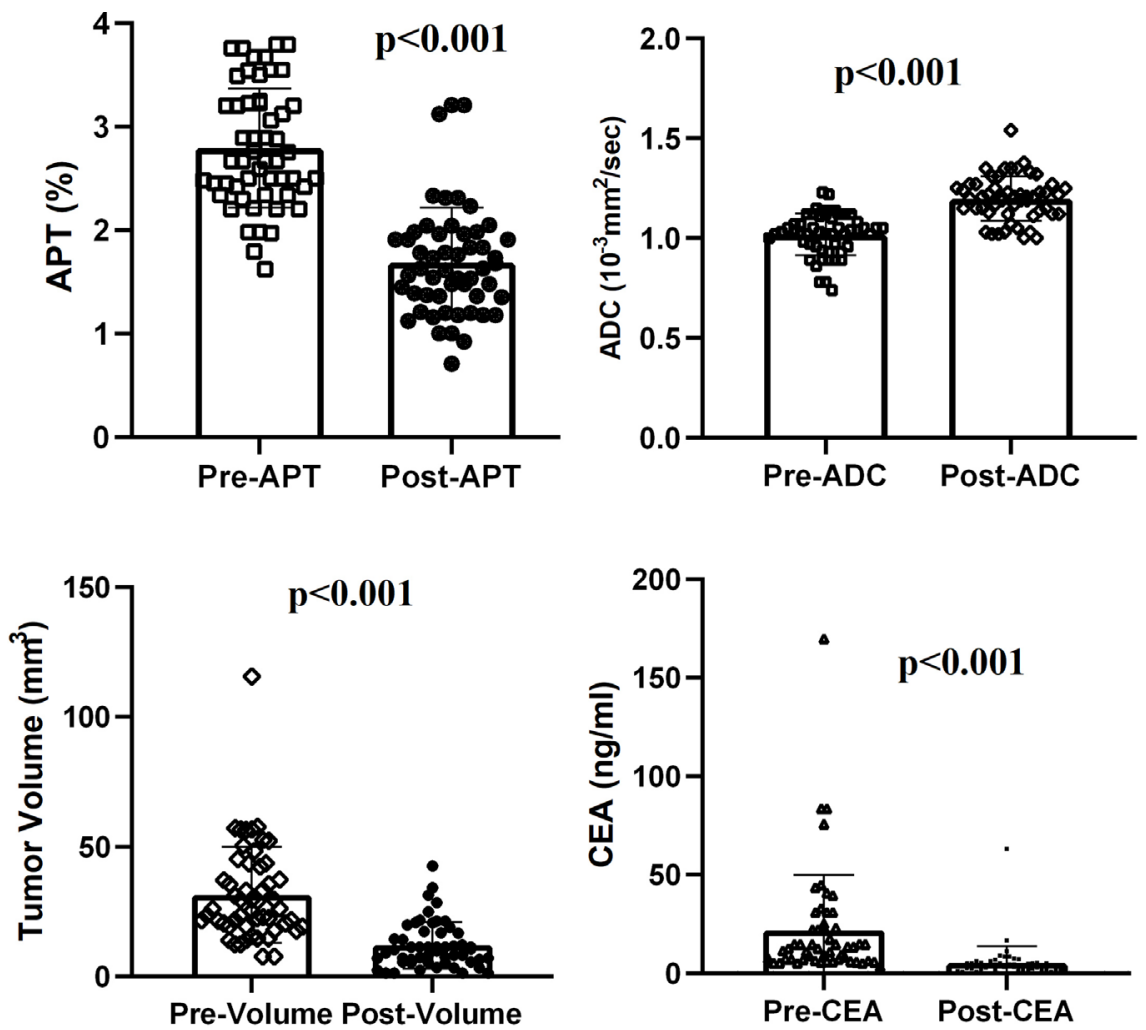

FIGURE 2 | Comparison of APT, ADC, tumor volume, and CEA level between pre- and post-NCRT.
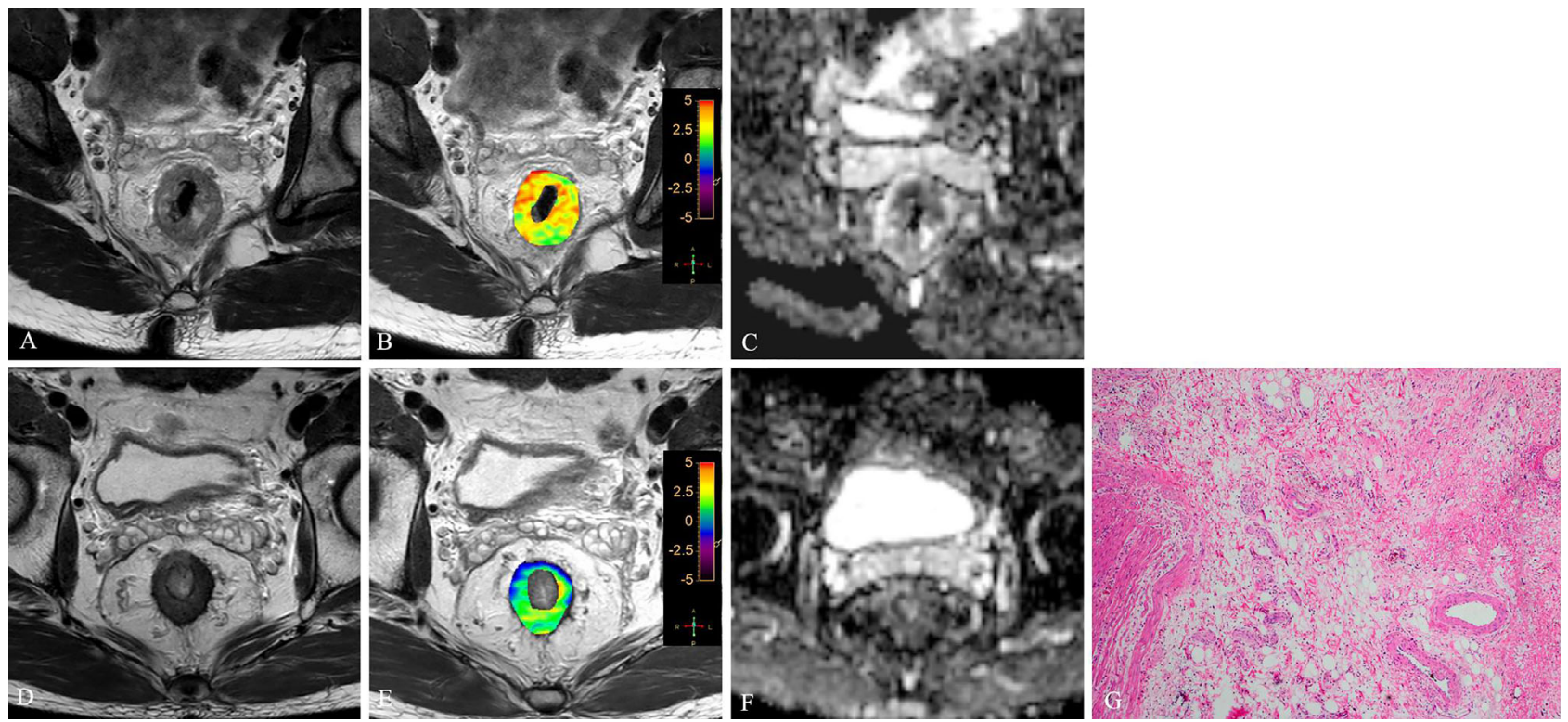

FIGURE 3 | T2WI (A, D), APTw (B, E) and ADC map (C, F) of a 63-year-old male with LARC before and after NCRT. At 8 weeks after NCRT, compared to the MR performed at baseline $(\mathbf{A}-\mathbf{C})$, the mean APTw SI decreased from $3.23 \%(\mathbf{B})$ to $1.54 \%$, the mean $\mathrm{ADC}$ value increased from $1.042 \times 10^{-3} \mathrm{~mm} / \mathrm{s}(\mathbf{C})$ to $1.112 \times 10^{-3}$ $\mathrm{mm}^{2} / \mathrm{s} \mathbf{( F )}$. Histopathological examination after surgery shows the degree of tumor regression is TRG 0 ( $\mathrm{H}$. E staining, $\left.\times 40, \mathbf{G}\right)$. 

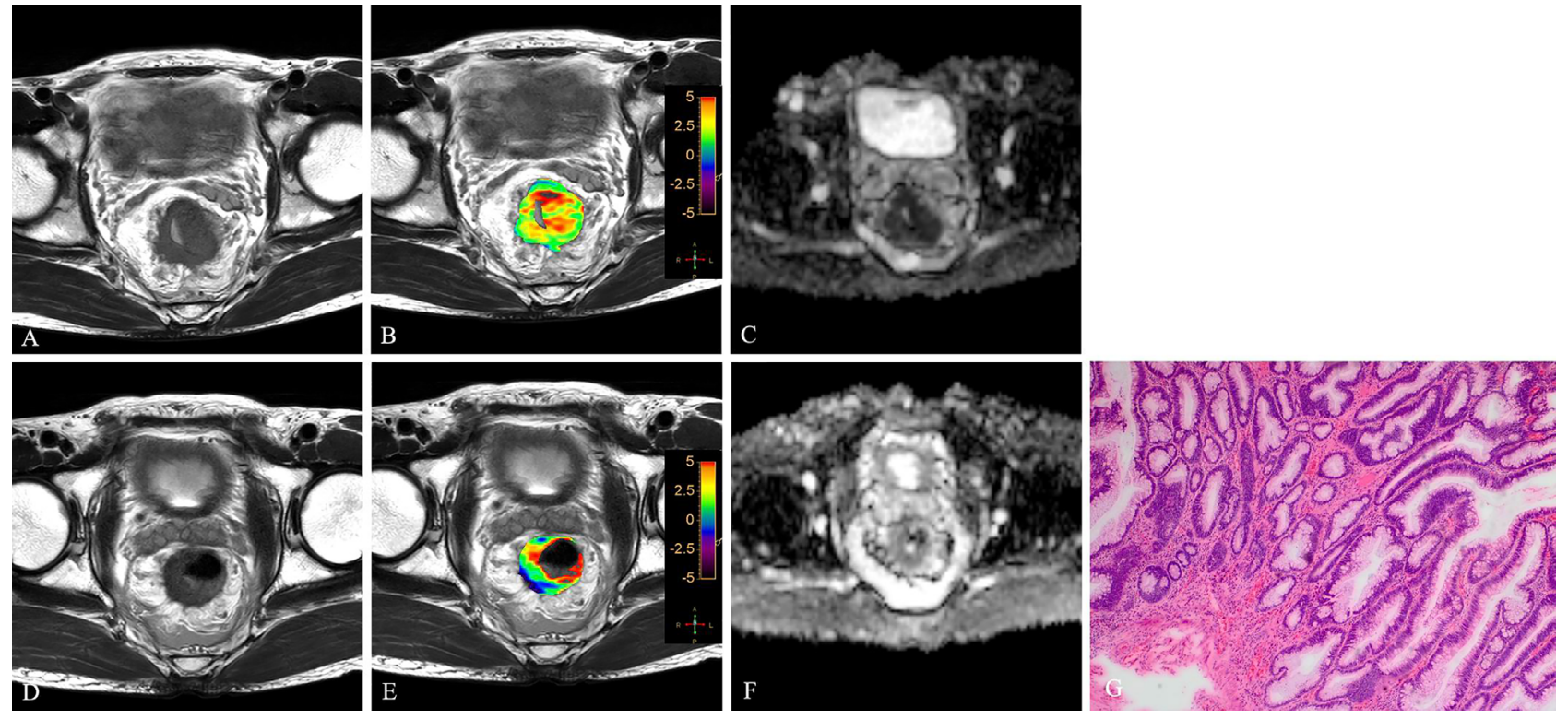

FIGURE 4 | T2WI (A, D), APTw (B, E) and ADC map (C, F) of a 56-year-old male with LARC before and after NCRT. At 8 weeks after NCRT, compared to the MR performed at baseline (A-C), the mean APTw SI decreased from $3.20 \%$ (B) to $1.614 \%$, the mean ADC value increased from $0.89 \times 10^{-3} \mathrm{~mm}^{2} / \mathrm{s}$ (C) to $1.234 \times 10^{-3}$ $\mathrm{mm}^{2} / \mathrm{s} \mathbf{( F )}$. Histopathological examination after surgery shows the degree of tumor regression is TRG $1(\mathrm{H}$. E staining, $\times 40, \mathbf{G})$.
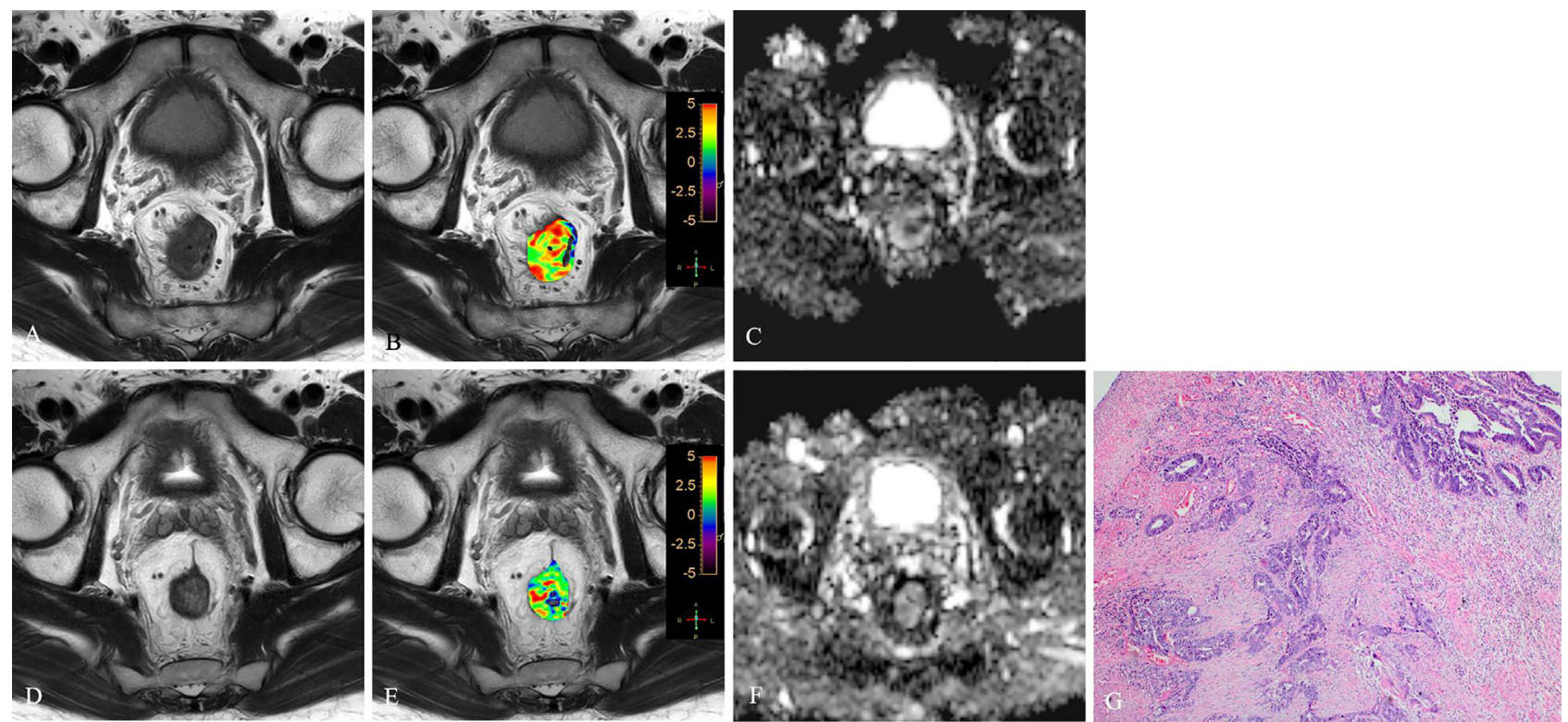

FIGURE 5 | T2WI (A, D), APTw (B, E) and ADC map (C, F) of a 51-year-old female with LARC before and after NCRT. At 6 weeks after NCRT, compared to the MR performed at baseline (A-C), the mean APTw SI decreased from $2.20 \%$ (B) to $1.783 \%$, the mean ADC value increased from $1.120 \times 10^{-3} \mathrm{~mm}^{2} / \mathrm{s}$ (C) to $1.205 \times 10^{-3}$ $\mathrm{mm}^{2} / \mathrm{s} \mathbf{( F )}$. Histopathological examination after surgery shows the degree of tumor regression is TRG $3(\mathrm{H}$. E staining, $\times 40, \mathbf{G})$.

NCRT are summarized in Table 4. The AUCs were 0.895, 0.800, $0.778,0.691,0.543$ and 0.680 for pre-APT\& pre-ADC, pre-APT, pre-ADC, $\triangle \mathrm{APT}, \triangle \mathrm{ADC}$ and $\Delta$ tumor volume, respectively. A combination of APT and ADC values before NCRT achieved a sensitivity of $85.29 \%$ and specificity of $89.47 \%$ for predicting a good response to NCRT, whereas PPV and NPV were $93.50 \%$ and $77.30 \%$, respectively (Table 4). Compare to pre-ADC, $\triangle \mathrm{ADC}$ and $\Delta$ tumor volume, pre-APT combined with pre- 

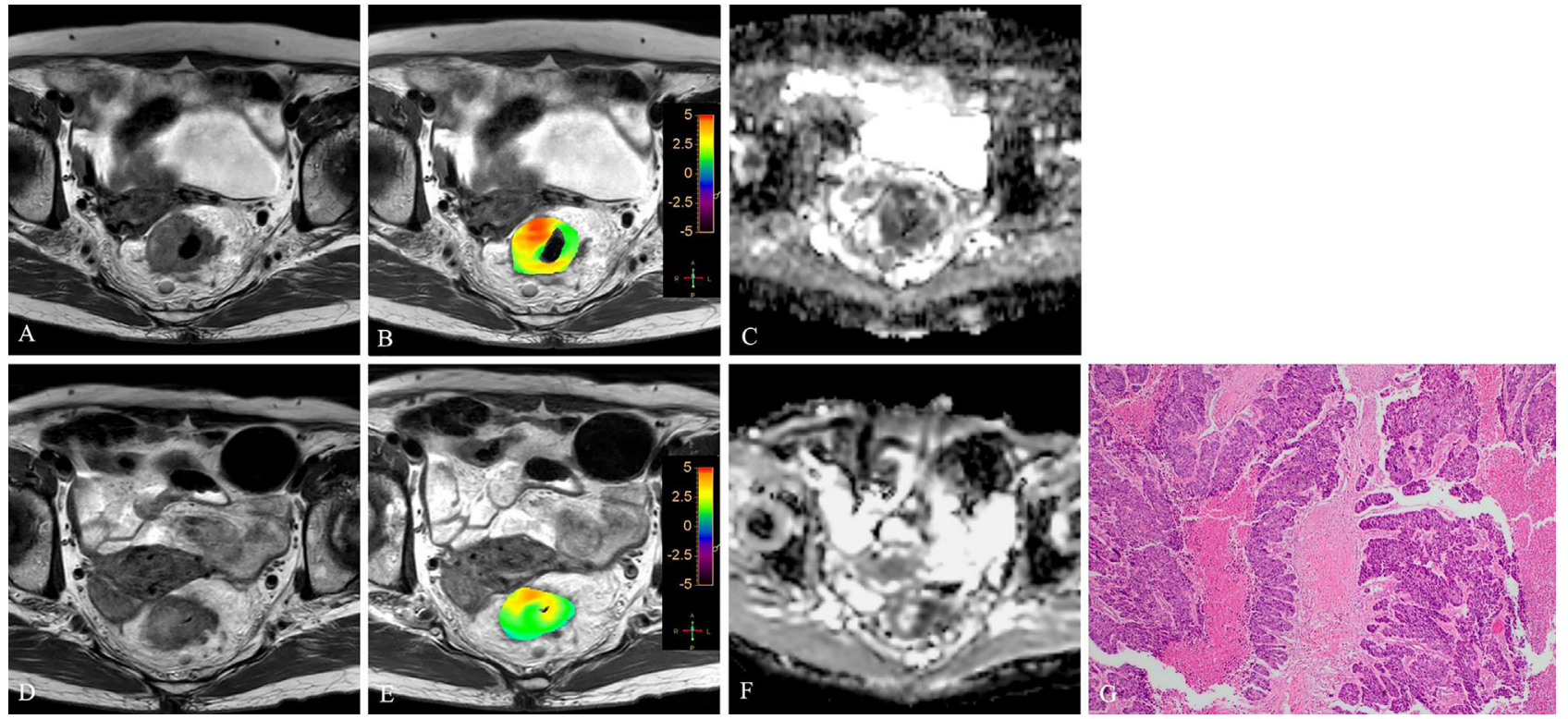

FIGURE 6 | T2WI (A, D), APTw (B, E) and ADC map (C, F) of a 47-year-old male with LARC before and after NCRT. At 6 weeks after NCRT, compared to the MR performed at baseline (A-C), the mean APTw SI decreased from $2.50 \%$ (B) to $1.83 \%$, the mean ADC value increased from $1.040 \times 10^{-3} \mathrm{~mm}^{2} / \mathrm{s}$ (C) to $1.245 \times 10^{-3}$ $\mathrm{mm}^{2} / \mathrm{s} \mathbf{( F )}$. Histopathological examination after surgery shows the degree of tumor regression is TRG $3(\mathrm{H}$. E staining, $\times 40, \mathbf{G})$

TABLE 3 | Comparison of variables between good and poor responders.

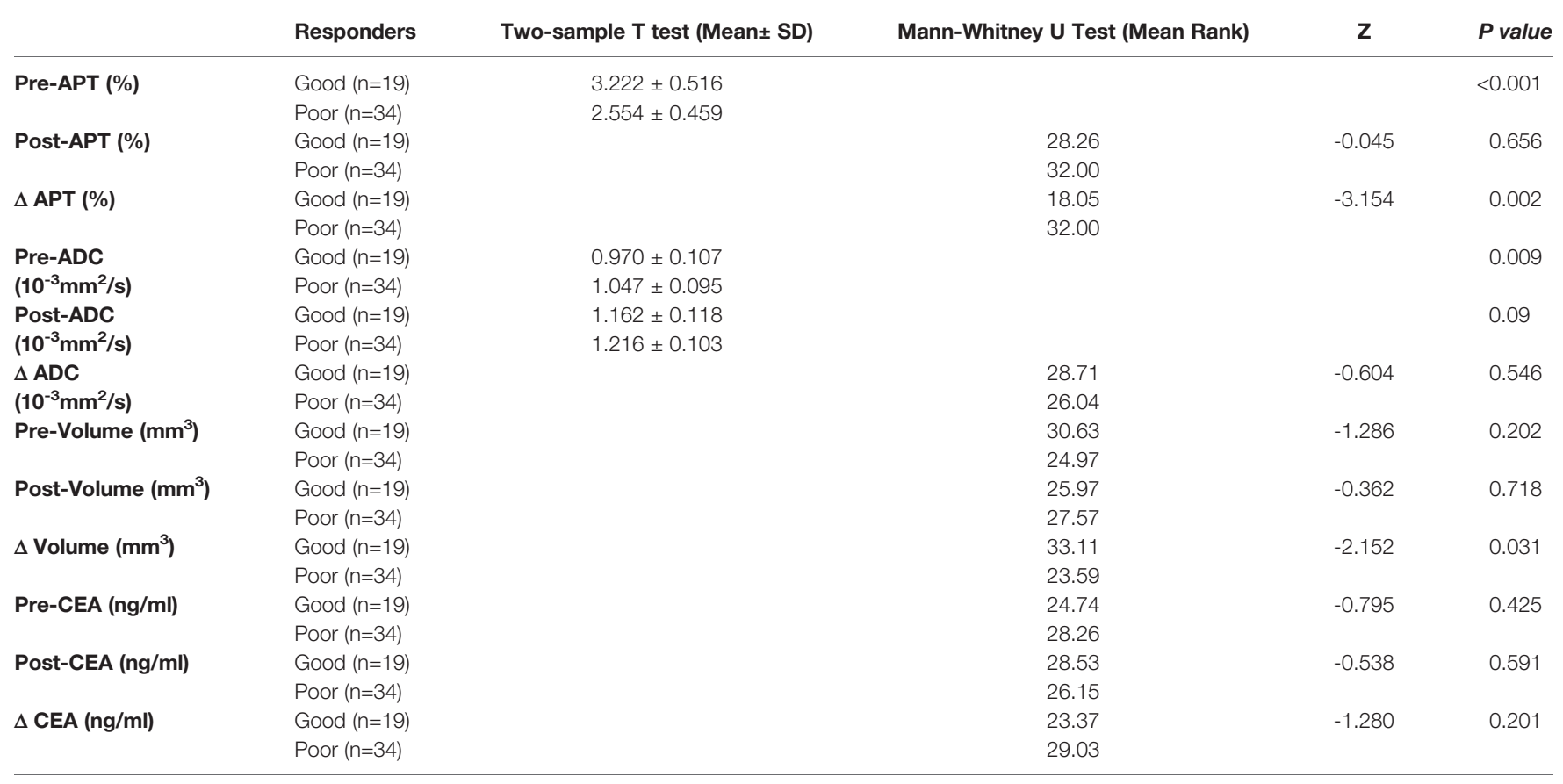

Data are means \pm standard deviations (normal distribution), mean rank (normal distribution failed). ADC values are given in $10^{-3} \mathrm{~mm}^{2} / \mathrm{sec}$, volume is given in $\mathrm{mm}{ }^{3}$, CEA level is given in $\mathrm{ng} / \mathrm{ml}$.

ADC showed greater diagnostic performance $(\mathrm{p}=0.019$, $\mathrm{p}<0.001$ and $\mathrm{p}=0.013$, respectively). However, there was no statistical difference among pre-APT \& ADC, pre-APT, and $\Delta$ APT (all p>0.05) (Figure 7).

\section{DISCUSSION}

In this study, we investigated the ability of APTw MRI combined with DWI to evaluate pathologic tumor down-staging and predict 
TABLE 4 | Performance of different MR parameters in predicting NCRT response in the patients with LARC.

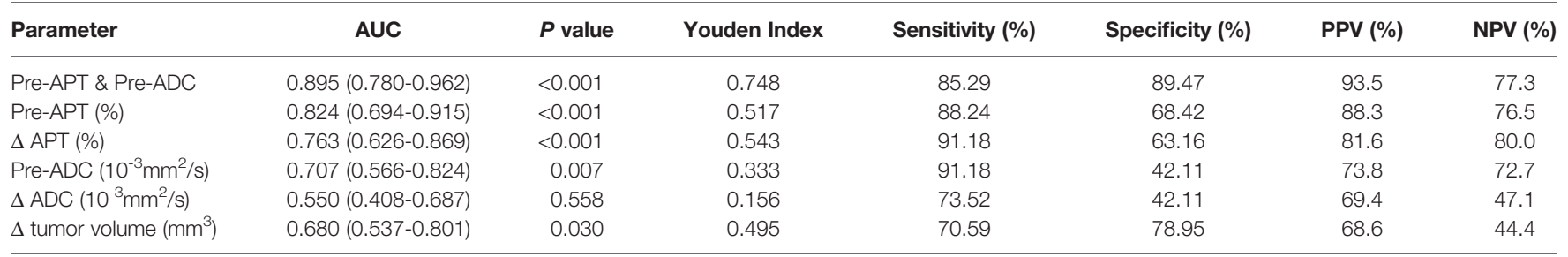

Data in parentheses are numerators and denominators and data in brackets are $95 \%$ confidence intervals. APT, amide proton transfer; ADC, apparent diffusion coefficient; AUC, area under curve; NPV, negative predictive value; PPV, positive predictive value.

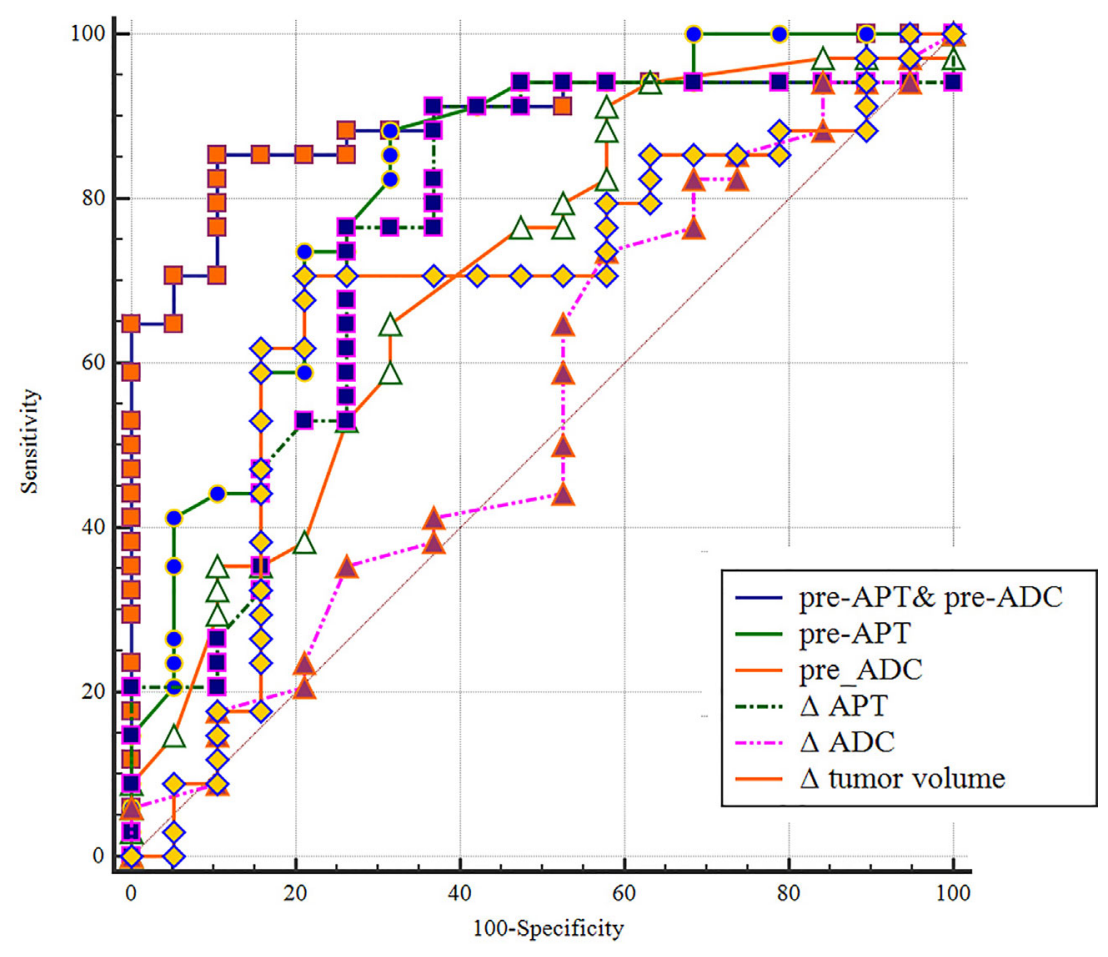

FIGURE 7 | A comparison of the diagnostic capability in predicting in discriminating good responders from poor responders between pre-APT \& pre-ADC, pre-APT, pre-ADC, $\triangle \mathrm{APT}, \triangle \mathrm{ADC}$ and $\Delta$ tumor volume. ROC analysis shows pre-APT combine with pre-ADC has a higher AUC (0.895) than the other parameters.

treatment responses after NCRT in rectal adenocarcinoma. After NCRT, all rectal tumors demonstrated significantly lower APT values and higher ADC values, as well as significantly smaller tumor volumes and lower CEA levels. APTw imaging is a new MRI contrast method based on CEST, using the signal of amide protons ( $\mathrm{NH}$ groups) contained in proteins and peptides. It has been suggested that endogenous mobile cytoplasmic proteins are the major source of APT signals (31). NCRT causes a series of pathological changes in rectal cancer, including cellular damage, tumor necrosis, local inflammatory reaction, and fibrosis replacing tumor glands (32), which leads to a lower content of proteins and peptides than in viable tumors and thus is expected to demonstrate a lower APTw signal. However, the increase in ADC after NCRT is due to increased diffusion of water molecules caused by cell damage, tumor necrosis, and extracellular edema.
When we compared these parameters between different therapeutic effect groups, we noted that the good responders demonstrated significantly higher APT values and lower ADC values before NCRT than the poor responders. Significantly higher $\triangle \mathrm{APT}$ values were also observed in patients with good responses. We suggest that the following factors might have contributed to this phenomenon. First, a high APT value for the tumor was associated with cellular proliferation and proved to be positively correlated with Ki67 expression level $(33,34)$, which is a cellular marker for cell proliferation and growth. Cellular proliferation is relevant to radiation response. Rapidly proliferating cells are more susceptible to NCRT-induced damage than quiescent cells because they have less time to repair the damage. Studies found that patients with higher Ki67 expression are associated with better response and downstaging 
in highly proliferative tumors, exhibiting a greater tumor regression grade response and $\mathrm{pCR}$ rate $(35,36)$. Second, tumor proliferation also depends on angiogenesis. Better-perfused tumors demonstrated higher proliferation capability, and blood could also generate sufficient CEST contrast. Studies have found high blood flow and permeability in good responders. Increased blood flow and enhanced microvascular permeability in the tumor bed provide not only better access for chemotherapy, but also less hypoxia-mediated radio-resistance, which contribute to good responses to NCRT (37). DCE-MRI of rectal cancer demonstrated that tumors with better perfusion showed greater nodal and tumor downstaging after radiotherapy than poorly perfused tumors (38). However, our result on APT changes after NCRT contrasted with that of Nishie et al., who found that pretherapeutic mean APTw SI of low-response group was significantly higher than that of the high-response group (29). This difference may be explained by the fact that Nieshi et al. used a different TRG grading criteria, which is according to necrosis or cytological changes of the tumor. In contrast, the TRG system in our study is based on the evaluation of residual tumor cells. Additionally, single-slice APT MR sequence in Nieshi et al.'s study may not reflect complete characteristics of the tumor.

DWI has been extensively used for prediction of response to neoadjuvant treatment in rectal cancer, and has been recommended in international clinical guidelines as a valuable adjunct to a restaging MRI protocol. All studies reported that tumor ADC values increased after NCRT, which is due to radiation-induced cellular damage and necrosis (39). However, results regarding the use of $\mathrm{ADC}$ in predicting the NCRT response have been inconsistent. Some studies demonstrated significantly lower pre-NCRT ADC values in the good responders (40-42), whereas Monguzzi L et al. found no benefit of pre- treatment ADC in assessing NCRT response (13). These conflicting results may be attributed to variations in DWI protocol, end-points, and variabilities in the patient selection criteria. Besides, it has been difficult to identify treatment response accurately based on DWI alone (39).

Additionally, tumor size and CEA level have proven to be independent predictors of pCR to NCRT in rectal cancer. Based on the results of systemic review and meta-analysis, small tumor size and low levels of pre-treatment CEA are associated with pCR or good response $(43,44)$. We found a significant reduction of tumor volume in good responders, which is in agreement with previous studies $(7,8)$. Although in the present study the good responders have lower CEA levels before NCRT, the difference is not significant.

Accurate early prediction of the response to NCRT would aid in the stratification of patients into optimal therapy managements and improve therapeutic outcomes in rectal cancer. Concerning discriminating tumor response to NCRT, we demonstrated that pre-APT combined with pre-ADC had the highest AUC (0.895) among all parameters. This combined parameter, with $85.29 \%$ sensitivity and $89.47 \%$ specificity, achieved greater efficacy than pre-ADC $(\mathrm{p}=0.029), \triangle \mathrm{ADC}(\mathrm{p}<0.001)$, pre-APT and $\triangle \mathrm{APT}$, although differences of AUCs between pre-APT\& pre-ADC, pre-APT, and $\triangle \mathrm{APT}$ were not statistically significant.
Our study has several limitations. The primary limitations were small sample size and lack of external validation steps. Second, we used the American Joint Committee on Cancer system for evaluating tumor regression. However, several grading systems are proposed for tumor regression, which may yield variable results owing to their different TRG components and grading criteria. Third, our study did not include evaluation of lymph node involvement, and it is known that presence of lymph node metastasis can be found in patients with pCR. Fourth, different chemotherapy regimens in our study may have an impact on the therapy efficacy. Lastly, our interpretation of APTw signal changes and tumor regression was based on the quantity of residual tumor cells and cytoplasmic protein. However, pathologic changes after NCRT are diverse and complex. Besides reduced tumor cellularity, variable histology changes after NCRT_including submucosal fibrosis, mucin pool formation, and calcification-could affect APT signal changes. Therefore, further research should be conducted to explain the biophysical sources of altered APT changes in rectal cancer after NCRT.

In conclusion, the combination of APTw MRI and DWI before NCRT holds potential in evaluating NCRT response of LARC, as is the capability to detect changes in cellular protein and cellularity density noninvasively, might provide additional information for clinical decision making in the management after NCRT.

\section{DATA AVAILABILITY STATEMENT}

The original contributions presented in the study are included in the article/Supplementary Material. Further inquiries can be directed to the corresponding author.

\section{ETHICS STATEMENT}

The studies involving human participants were reviewed and approved by The Second Affiliated Hospital, Guangzhou University of Chinese Medicine. The patients/participants provided their written informed consent to participate in this study.

\section{AUTHOR CONTRIBUTIONS}

The authors confirm contribution to the paper as follows: study conception and design: XL, WC, and LL. Data collection: QW, $\mathrm{JP}$, and $\mathrm{SH}$. Analysis and interpretation of results: WC, LM, and YY. Draft manuscript preparation: WC, BL, and XL. All authors contributed to the article and approved the submitted version.

\section{SUPPLEMENTARY MATERIAL}

The Supplementary Material for this article can be found online at: https://www.frontiersin.org/articles/10.3389/fonc.2021.698427/ full\#supplementary-material 


\section{REFERENCES}

1. Oronsky B, Reid T, Larson C, Knox SJ. Locally Advanced Rectal Cancer: The Past, Present, and Future. Semin Oncol (2020) 47(1):85-92. doi: 10.1053/ j.seminoncol.2020.02.001

2. Petrelli F, Trevisan F, Cabiddu M, Sgroi G, Bruschieri L, Rausa E, et al. Total Neoadjuvant Therapy in Rectal Cancer: A Systematic Review and MetaAnalysis of Treatment Outcomes. Ann Surg (2020) 271(3):440-8. doi: 10.1097/SLA.0000000000003471

3. Dossa F, Chesney TR, Acuna SA, Baxter NN. A Watch-and-Wait Approach for Locally Advanced Rectal Cancer After a Clinical Complete Response Following Neoadjuvant Chemoradiation: A Systematic Review and MetaAnalysis. Lancet Gastroenterol Hepatol (2017) 2(7):501-13. doi: 10.1016/ S2468-1253(17)30074-2

4. Riesco-Martinez MC, Fernandez-Martos C, Gravalos-Castro C, EspinosaOlarte P, La Salvia A, Robles-Diaz L, et al. Impact of Total Neoadjuvant Therapy vs. Standard Chemoradiotherapy in Locally Advanced Rectal Cancer: A Systematic Review and Meta-Analysis of Randomized Trials. Cancers (2020) 12(12):3655. doi: 10.3390/cancers 12123655

5. Kalisz KR, Enzerra MD, Paspulati RM. MRI Evaluation of the Response of Rectal Cancer to Neoadjuvant Chemoradiation Therapy. Radiographics: Rev Publ Radiol Soc North Am Inc (2019) 39(2):538-56. doi: 10.1148/rg.2019180075

6. Patel UB, Taylor F, Blomqvist L, George C, Evans H, Tekkis P, et al. Magnetic Resonance Imaging-Detected Tumor Response for Locally Advanced Rectal Cancer Predicts Survival Outcomes: MERCURY Experience. J Clin Oncol: Off J Am Soc Clin Oncol (2011) 29(28):3753-60. doi: 10.1200/JCO.2011.34.9068

7. Curvo-Semedo L, Lambregts DM, Maas M, Thywissen T, Mehsen RT, Lammering G, et al. Rectal Cancer: Assessment of Complete Response to Preoperative Combined Radiation Therapy With ChemotherapyConventional MR Volumetry Versus Diffusion-Weighted MR Imaging. Radiology (2011) 260(3):734-43. doi: 10.1148/radiol.11102467

8. Kang JH, Kim YC, Kim H, Kim YW, Hur H, Kim JS, et al. Tumor Volume Changes Assessed by Three-Dimensional Magnetic Resonance Volumetry in Rectal Cancer Patients After Preoperative Chemoradiation: The Impact of the Volume Reduction Ratio on the Prediction of Pathologic Complete Response. Int J Radiat Oncol Biol Phys (2010) 76(4):1018-25. doi: 10.1016/j.ijrobp. 2009.03.066

9. Neri E, Guidi E, Pancrazi F, Castagna M, Castelluccio E, Balestri R, et al. MRI Tumor Volume Reduction Rate vs Tumor Regression Grade in the PreOperative Re-Staging of Locally Advanced Rectal Cancer After ChemoRadiotherapy. Eur J Radiol (2015) 84(12):2438-43. doi: 10.1016/ j.ejrad.2015.08.008

10. Fiorino C, Gumina C, Passoni P, Palmisano A, Broggi S, Cattaneo GM, et al. A TCP-Based Early Regression Index Predicts the Pathological Response in Neo-Adjuvant Radio-Chemotherapy of Rectal Cancer. Radiother Oncol: J Eur Soc Ther Radiol Oncol (2018) 128(3):564-8. doi: 10.1016/j.radonc.2018.06.019

11. Fiorino C, Passoni P, Palmisano A, Gumina C, Cattaneo GM, Broggi S, et al. Accurate Outcome Prediction After Neo-Adjuvant Radio-Chemotherapy for Rectal Cancer Based on a TCP-Based Early Regression Index. Clin Trans Radiat Oncol (2019) 19:12-6. doi: 10.1016/j.ctro.2019.07.001

12. Lambregts DMJ, van Heeswijk MM, Delli Pizzi A, van Elderen SGC, Andrade L, Peters N, et al. Diffusion-Weighted MRI to Assess Response to Chemoradiotherapy in Rectal Cancer: Main Interpretation Pitfalls and Their Use for Teaching. Eur Radiol (2017) 27(10):4445-54. doi: 10.1007/ s00330-017-4830-Z

13. Nie K, Shi L, Chen Q, Hu X, Jabbour SK, Yue N, et al. Rectal Cancer: Assessment of Neoadjuvant Chemoradiation Outcome Based on Radiomics of Multiparametric MRI. Clin Cancer Res: an Off J Am Assoc Cancer Res (2016) 22(21):5256-64. doi: 10.1158/1078-0432.CCR-15-2997

14. Dijkhoff RAP, Beets-Tan RGH, Lambregts DMJ, Beets GL, Maas M. Value of DCE-MRI for Staging and Response Evaluation in Rectal Cancer: A Systematic Review. Eur J Radiol (2017) 95:155-68. doi: 10.1016/ j.ejrad.2017.08.009

15. Intven M, Reerink O, Philippens ME. Dynamic Contrast Enhanced MR Imaging for Rectal Cancer Response Assessment After Neo-Adjuvant Chemoradiation. J Magn Reson Imaging: JMRI (2015) 41(6):1646-53. doi: 10.1002/jmri. 24718
16. Shaish H, Aukerman A, Vanguri R, Spinelli A, Armenta P, Jambawalikar S, et al. Radiomics of MRI for Pretreatment Prediction of Pathologic Complete Response, Tumor Regression Grade, and Neoadjuvant Rectal Score in Patients With Locally Advanced Rectal Cancer Undergoing Neoadjuvant Chemoradiation: An International Multicenter Study. Eur Radiol (2020) 30 (11):6263-73. doi: 10.1007/s00330-020-06968-6

17. Horvat N, Veeraraghavan H, Khan M, Blazic I, Zheng J, Capanu M, et al. MR Imaging of Rectal Cancer: Radiomics Analysis to Assess Treatment Response After Neoadjuvant Therapy. Radiology (2018) 287(3):833-43. doi: 10.1148/ radiol.2018172300

18. Zhang XY, Wang L, Zhu HT, Li ZW, Ye M. Predicting Rectal Cancer Response to Neoadjuvant Chemoradiotherapy Using Deep Learning of Diffusion Kurtosis MRI. Radiology (2020) 296(1):56-64. doi: 10.1148/ radiol.2020190936

19. Peeken JC, Bernhofer M, Wiestler B, Goldberg T, Cremers D, Rost B, et al. Radiomics in Radiooncology - Challenging the Medical Physicist. Physica Med: PM: an Int J Devoted to Appl Phys to Med Biol: Off J Ital Assoc Biomed Phys (AIFB) (2018) 48:27-36. doi: 10.1016/j.ejmp.2018.03.012

20. Ching T, Himmelstein DS, Beaulieu-Jones BK. Opportunities and Obstacles for Deep Learning in Biology and Medicine. J R Soc Interface (2018) 15 (141):20170387. doi: 10.1098/rsif.2017.0387

21. Zhou J, Heo HY, Knutsson L, van Zijl PCM, Jiang S. APT-Weighted MRI: Techniques, Current Neuro Applications, and Challenging Issues. J Magn Reson Imaging: JMRI (2019) 50(2):347-64. doi: 10.1002/jmri.26645

22. Togao O, Yoshiura T, Keupp J, Hiwatashi A, Yamashita K, Kikuchi K, et al. Amide Proton Transfer Imaging of Adult Diffuse Gliomas: Correlation With Histopathological Grades. Neuro-Oncology (2013) 16(3):441-8. doi: 10.1093/ neuonc/not158

23. Chen W, Li L, Yan Z, Hu S, Feng J, Liu G, et al. Three-Dimension Amide Proton Transfer MRI of Rectal Adenocarcinoma: Correlation With Pathologic Prognostic Factors and Comparison With Diffusion Kurtosis Imaging. Eur Radiol (2021) 31(5):3286-96. doi: 10.1007/s00330-020-07397-1

24. Suh CH, Park JE, Jung SC, Choi CG, Kim SJ, Kim HS. Amide Proton TransferWeighted MRI in Distinguishing High- and Low-Grade Gliomas: A Systematic Review and Meta-Analysis. Neuroradiology (2019) 61(5):525-34. doi: 10.1007/s00234-018-02152-2

25. Mehrabian H, Desmond KL, Soliman H, Sahgal A, Stanisz GJ. Differentiation Between Radiation Necrosis and Tumor Progression Using Chemical Exchange Saturation Transfer. Clin Cancer Res: an Off J Am Assoc Cancer Res (2017) 23(14):3667-75. doi: 10.1158/1078-0432.CCR-16-2265

26. Park JE, Kim HS, Park KJ, Kim SJ, Kim JH, Smith SA. Pre- and Posttreatment Glioma: Comparison of Amide Proton Transfer Imaging With MR Spectroscopy for Biomarkers of Tumor Proliferation. Radiology (2016) 278 (2):514-23. doi: 10.1148/radiol.2015142979

27. Park KJ, Kim HS, Park JE, Shim WH, Kim SJ, Smith SA. Added Value of Amide Proton Transfer Imaging to Conventional and Perfusion MR Imaging for Evaluating the Treatment Response of Newly Diagnosed Glioblastoma. Eur Radiol (2016) 26(12):4390-403. doi: 10.1007/s00330-016-4261-2

28. Zhou J, Tryggestad E, Wen Z, Lal B, Zhou T, Grossman R, et al. Differentiation Between Glioma and Radiation Necrosis Using Molecular Magnetic Resonance Imaging of Endogenous Proteins and Peptides. Nat Med (2011) 17(1):130-4. doi: 10.1038/nm.2268

29. Nishie A, Asayama Y, Ishigami K, Ushijima Y, Takayama Y, Okamoto D, et al. Amide Proton Transfer Imaging to Predict Tumor Response to Neoadjuvant Chemotherapy in Locally Advanced Rectal Cancer. J Gastroenterol Hepatol (2019) 34(1):140-6. doi: 10.1111/jgh.14315

30. Amin MB ES, Greene F, Byrd DR, Brookland RK, Washington MK. Ajcc Cancer Staging Manual. 8th ed. New York (NY): Springer (2016).

31. Zhou J, Lal B, Wilson DA, Laterra J, van Zijl PC. Amide Proton Transfer (APT) Contrast for Imaging of Brain Tumors. Magn Reson Med (2003) 50 (6):1120-6. doi: 10.1002/mrm.10651

32. Hav M, Libbrecht L, Ferdinande L, Geboes K, Pattyn P, Cuvelier CA. Pathologic Assessment of Rectal Carcinoma After Neoadjuvant Radio (Chemo)Therapy: Prognostic Implications. BioMed Res Int (2015) 2015:574540. doi: 10.1155/2015/574540

33. Yu H, Wen X, Wu P, Chen Y, Zou T, Wang X, et al. Can Amide Proton Transfer-Weighted Imaging Differentiate Tumor Grade and Predict Ki-67 
Proliferation Status of Meningioma? Eur Radiol (2019) 29(10):5298-306. doi: 10.1007/s00330-019-06115-w

34. Chen Y, Li X, Song Y, Zhu X, Zhao J, Yan X, et al. The Diagnostic Efficacy of Amide Proton Transfer Imaging in Grading Gliomas and Predicting Tumor Proliferation. Neuroreport (2019) 30(2):139-44. doi: 10.1097/WNR.0000000000001174

35. Kim NK, Park JK, Lee KY, Yang WI, Yun SH, Sung J, et al. P53, BCL-2, and Ki-67 Expression According to Tumor Response After Concurrent Chemoradiotherapy for Advanced Rectal Cancer. Ann Surg Oncol (2001) 8 (5):418-24. doi: 10.1007/s10434-001-0418-5

36. Melling N, Kowitz CM, Simon R, Bokemeyer C, Terracciano L, Sauter G, et al. High Ki67 Expression Is an Independent Good Prognostic Marker in Colorectal Cancer. J Clin Pathol (2016) 69(3):209-14. doi: 10.1136/ jclinpath-2015-202985

37. Höckel M, Vaupel P. Biological Consequences of Tumor Hypoxia. Semin Oncol (2001) 28(2 Suppl 8):36-41. doi: 10.1053/sonc.2001.25392

38. Zahra MA, Hollingsworth KG, Sala E, Lomas DJ, Tan LT. Dynamic ContrastEnhanced MRI as a Predictor of Tumour Response to Radiotherapy. Lancet Oncol (2007) 8(1):63-74. doi: 10.1016/S1470-2045(06)71012-9

39. Kim SH, Lee JM, Hong SH, Kim GH, Lee JY, Han JK, et al. Locally Advanced Rectal Cancer: Added Value of Diffusion-Weighted MR Imaging in the Evaluation of Tumor Response to Neoadjuvant Chemo- and Radiation Therapy. Radiology (2009) 253(1):116-25. doi: 10.1148/radiol.2532090027

40. Jung SH, Heo SH, Kim JW, Jeong YY, Shin SS, Soung MG, et al. Predicting Response to Neoadjuvant Chemoradiation Therapy in Locally Advanced Rectal Cancer: Diffusion-Weighted 3 Tesla MR Imaging. J Magn Reson Imaging: JMRI (2012) 35(1):110-6. doi: 10.1002/jmri.22749

41. Chen YG, Chen MQ, Guo YY, Li SC, Wu JX, Xu BH. Apparent Diffusion Coefficient Predicts Pathology Complete Response of Rectal Cancer Treated
With Neoadjuvant Chemoradiotherapy. PloS One (2016) 11(4):e0153944. doi: 10.1371/journal.pone.0153944

42. Jacobs L, Intven M, van Lelyveld N, Philippens M, Burbach M, Seldenrijk K, et al. Diffusion-Weighted MRI for Early Prediction of Treatment Response on Preoperative Chemoradiotherapy for Patients With Locally Advanced Rectal Cancer: A Feasibility Study. Ann Surg (2016) 263(3):522-8. doi: 10.1097/ SLA. 0000000000001311

43. Lee YC, Hsieh CC, Chuang JP. Prognostic Significance of Partial Tumor Regression After Preoperative Chemoradiotherapy for Rectal Cancer: A MetaAnalysis. Dis Colon Rectum (2013) 56(9):1093-101. doi: 10.1097/ DCR.0b013e318298e36b

44. Colloca G, Venturino A, Vitucci P. Pre-Treatment Carcinoembryonic Antigen and Outcome of Patients With Rectal Cancer Receiving Neo-Adjuvant Chemo-Radiation and Surgical Resection: A Systematic Review and MetaAnalysis. Med Oncol (Northwood London England) (2017) 34(10):177. doi: 10.1007/s12032-017-1037-8

Conflict of Interest: The authors declare that the research was conducted in the absence of any commercial or financial relationships that could be construed as a potential conflict of interest.

Copyright $\odot 2021$ Chen, Mao, Li, Wei, Hu, Ye, Feng, Liu and Liu. This is an openaccess article distributed under the terms of the Creative Commons Attribution License (CC BY). The use, distribution or reproduction in other forums is permitted, provided the original author(s) and the copyright owner(s) are credited and that the original publication in this journal is cited, in accordance with accepted academic practice. No use, distribution or reproduction is permitted which does not comply with these terms. 